\title{
SPATIO-TEMPORAL REGULARIZATION OF FLOW-FIELDS
}

\author{
Emrah Bostan ${ }^{\star}$ Orestis Vardoulis ${ }^{\S}$ Davide Piccini ${ }^{*}$, Pouya Dehghani Tafti, Nikolaos Stergiopulos $\$$, and Michael Unser ${ }^{\star}$ \\ ${ }^{\star}$ Biomedical Imaging Group, EPFL, Lausanne, Switzerland \\ ${ }^{\S}$ Laboratory of Hemodynamics and Cardiovascular Technology, EPFL, Lausanne, Switzerland \\ ${ }^{*}$ Siemens Schweiz AG, Healthcare Sector, Lausanne, Switzerland \\ ${ }^{\ddagger}$ Center for Biomedical Imaging (CIBM), Lausanne, Switzerland
}

\begin{abstract}
We introduce a novel variational framework for the regularized reconstruction of time-resolved volumetric flow fields. Our objective functional takes the physical characteristics of the underlying flow into account in both the spatial and the temporal domains. For an efficient minimization of the objective functional, we apply a proximal-splitting algorithm and perform parallel computations. To demonstrate the utility of our variational method, we first denoise a simulated flow-field in the human aorta and show that our method outperforms spatial-only regularization in terms of signal-to-noise ratio (SNR). We then apply the scheme to a real 3D+time phase-contrast MRI dataset and obtain high-quality visualizations.
\end{abstract}

Index Terms - Spatio-temporal regularization, flow fields, vector fields, 4D flow MRI, flow-sensitive MRI, phase-contrast MRI, denoising, proximal splitting, curl, divergence, invariance.

\section{INTRODUCTION}

Recent methodological improvements in phase-contrast (PC) MRI enable the acquisition of time-dependent blood flow with full volumetric coverage [1]. In a noninvasive manner, PC MRI is able to measure the blood flow in a specific vascular region of interest. This makes PC MRI (also termed flow-sensitive MRI) highly suitable for designing diagnostic and treatment strategies for cardiovascular diseases [2]. For instance, PC MRI measurements can be used to assess the generation of complex flow phenomena and unfavorable shear stress along the aged or diseased central arteries. These data acquired in vivo can be also combined with 1D or 3D computational fluid dynamics to develop methods for evaluating surgical outcomes in the aorta [3]. As for all MR recordings, the quality of PC MRI measurements are degraded by noise. This degradation becomes more severe if the acquisition time is shortened, which is however desirable for increased temporal resolution. Several other sources of error such as background phase contributions from eddy-currents or velocity aliasing can also distort flow maps [1]. Furthermore, a priori known physical properties of the flow are not automatically enforced in the raw, noisy acquisitions.

Following these reasonings, we shall consider a variational method that takes the form of the optimization problem

$$
\mathbf{f}^{\star}=\underset{\mathbf{f}}{\operatorname{argmin}} \mathcal{D}(\mathbf{f} ; \mathbf{y})+\sum_{i} \mathcal{R}_{i}(\mathbf{f}),
$$

where the data fidelity term $\mathcal{D}$ keeps the reconstructed field $\mathbf{f}^{\star}$ close to the measured flow $\mathbf{y}$ and where each regularization functional (or regularizer) $\mathcal{R}_{i}$ imposes certain characteristics on $\mathbf{f}^{\star}$.

This work was partially supported by the Leenaards and Louis-Jeannet foundations and by the European Commission under Grant ERC-2010-AdG 267439-FUN-SP.
When specifying such an algorithm, a guiding principle is to impose physically sound constraints on the solution. In particular, it is highly desirable that the solution remains invariant to certain coordinate transformations such as translation, rotation, and scaling. The curl and divergence operators are particularly well-suited for that purpose. They have been used by different authors for flow-field regularization. For instance, Suter and Chen [4] and Arigovindan et al. [5] have considered them in the context of quadratic $\left(\mathrm{L}_{2}\right)$ regularization. Tafti and Unser [6] and Tafti et al. [7] have showed that the discontinuities at the flow boundaries are better preserved by switching from an $\mathrm{L}_{2}$-norm to an $\mathrm{L}_{1}$-norm regularization.

While the said formulations are capable of imposing valid constraints on the solution, they are fundamentally limited to spatial regularization of 2D and/or 3D flows. They are not truly suited for dynamic-flow imaging applications. In the present paper, based on our previous approaches on flow-field reconstruction $[6,7,8]$, we introduce a variational method for time-dependent volumetric flow-field data.

The main contributions of this work are:

- The formulation of a spatio-temporal regularization method that is appropriate to the tasks of denoising and enhancement of 3D+time flow-fields. Our method involves curl and divergence operators for the spatial regularization and assumes that the underlying flow is varying smoothly in time.

- The derivation of a reconstruction algorithm, based on proximal-splitting, that decomposes the optimization into sub-problems that are solved in parallel.

- The application of the resulting scheme to the problem of flow-field denoising and visual enhancement. We show that our algorithm achieves better denoising performance than its spatial-only counterpart. We further apply the method to a real PC MRI data of blood flow in the human aorta and obtain improved pathline visualization of the flow.

\section{VARIATIONAL MODEL}

A time-dependent volumetric flow-field is represented by the vector function $\mathbf{f}(\mathbf{s}, t)=\left(f_{1}(\mathbf{s}, t), f_{2}(\mathbf{s}, t), f_{3}(\mathbf{s}, t)\right)$ over some bounded spatio-temporal domain $\Omega_{\mathbf{s}} \times \Omega_{t} \subset \mathbb{R}^{3} \times \mathbb{R} ; \mathbf{s} \in \Omega_{\mathbf{s}}$ denotes the spatial coordinates and $t \in \Omega_{t}$ the temporal one.

\subsection{Continuous-Domain Formulation}

In the continuous setting, the generic form of the regularization functional is given by

$$
\mathcal{R}(\mathbf{f})=\lambda \int_{\mathbb{R}} \int_{\mathbb{R}^{3}} \phi(\mathrm{R}\{\mathbf{f}\}(\mathbf{s}, t)) \mathrm{d} \mathbf{s} \mathrm{d} t,
$$


where $\mathrm{R}$ is the regularization operator (scalar- or vector-valued), $\phi: \mathbb{R}^{N} \rightarrow \mathbb{R}$ is a potential function ( $N$ depends on the codomain of $\mathrm{R}\{\mathbf{f}\})$, and $\lambda>0$ is the regularization parameter.

As for the choice of suitable spatial regularization operators, we shall rely on first-order differential operators such as div and curl as justified by Proposition 1 below. Considering the short transition space between the artery walls, we choose $\phi(\cdot)=|\cdot|$ denoting the absolute value or the magnitude depending on its argument. This potential function has been shown to be well-suited for preserving abrupt flow transitions at the boundaries [6]. Accordingly, we define the regularizers

$$
\begin{aligned}
& \mathcal{R}_{1}(\mathbf{f})=\lambda_{c} \int_{\mathbb{R}} \int_{\mathbb{R}^{3}}|\operatorname{curl}\{\mathbf{f}(\cdot, t)\}(\mathbf{s})| \mathrm{d} \mathbf{s} \mathrm{d} t, \\
& \mathcal{R}_{2}(\mathbf{f})=\lambda_{d} \int_{\mathbb{R}} \int_{\mathbb{R}^{3}}|\operatorname{div}\{\mathbf{f}(\cdot, t)\}(\mathbf{s})| \mathrm{d} \mathbf{s} \mathrm{d} t .
\end{aligned}
$$

In effect, (3) and (4) allow one to penalize the total curl and/or divergence - thus, the irrotational and incompressible behavior-of the time-resolved flow.

In the temporal dimension, we assume that the flow is "smooth" and take $\phi(\cdot)=|\cdot|^{2}$. We then define

$$
\mathcal{R}_{3}(\mathbf{f})=\lambda_{t} \int_{\mathbb{R}} \int_{\mathbb{R}^{3}}\left|\partial_{t} \mathbf{f}(\mathbf{s}, t)\right|^{2} \mathrm{~d} \mathbf{s} \mathrm{d} t,
$$

where $\partial_{t}$ is the time derivative. Note that our assumption remains valid for laminar flows. Moreover, as explained in Nichols et al. [9], flow velocity waveforms in the ascending and descending aorta are varying smoothly through the cardiac cycle.

Proposition 1. Let $\mathcal{R}_{1}, \mathcal{R}_{2}, \mathcal{R}_{3}$ be defined as in (3), (4), and (5), respectively. Then, each of these terms is invariant under scaling (up to a multiplicative factor) and translation in space and time, and rotation-invariant in space, where the rotation of a vector field by some orthogonal matrix $\xi$ is given by $\mathbf{f} \mapsto \xi^{\mathrm{T}} \mathbf{f}(\xi \cdot t)$.

Proof. Translation- and scale-invariance both in time and in space are obvious (derivatives are translation- and scale-invariant and the integrals are translation-invariant and linear). To prove rotationinvariance, we consider equivalent tensor definition of curl (see [6]). We note that in Fourier domain,

$$
\begin{aligned}
\mathcal{F}\{\operatorname{div} \mathbf{f}\} & =\mathrm{j} \boldsymbol{\omega}_{\mathbf{s}}^{\mathrm{T}} \hat{\mathbf{f}} \\
\mathcal{F}\{\mathbf{c u r l} \mathbf{f}\} & =\mathrm{j} \boldsymbol{\omega}_{\mathbf{s}} \hat{\mathbf{f}}^{\mathrm{T}}-\hat{\mathbf{f}} \mathbf{j} \boldsymbol{\omega}_{\mathbf{s}}^{\mathrm{T}} \\
\mathcal{F}\left\{\partial_{t} \mathbf{f}\right\} & =\mathrm{j} \omega_{t} \hat{\mathbf{f}}
\end{aligned}
$$

For rotation by a matrix $\xi$ we have $\left(R_{\xi}\right.$ is the rotation operator):

$$
\begin{aligned}
\mathcal{F}\left\{\operatorname{div} R_{\xi} \mathbf{f}\right\} & =j \boldsymbol{\omega}_{\mathbf{s}}^{\mathrm{T}} \xi^{\mathrm{T}} \hat{\mathbf{f}}\left(\xi \boldsymbol{\omega}_{\mathbf{s}}, \omega_{t}\right)=\mathrm{j}\left(\xi \boldsymbol{\omega}_{\mathbf{s}}\right)^{\mathrm{T}} \hat{\mathbf{f}}\left(\xi \boldsymbol{\omega}_{\mathbf{s}}, \omega_{t}\right) \\
& =\mathcal{F}\left\{\mathrm{R}_{\xi} \operatorname{div} \mathbf{f}\right\} . \\
\mathcal{F}\left\{\operatorname{curl} \mathrm{R}_{\xi} \mathbf{f}\right\} & =\mathrm{j} \boldsymbol{\omega}_{\mathbf{s}} \hat{\mathbf{f}}^{\mathrm{T}}\left(\xi \boldsymbol{\omega}_{\mathbf{s}}, \omega_{t}\right) \xi-\xi^{\mathrm{T}} \hat{\mathbf{f}}\left(\xi \boldsymbol{\omega}_{\mathbf{s}}, \omega_{t}\right) \mathrm{j} \boldsymbol{\omega}_{\mathbf{s}}^{\mathrm{T}} \\
& =\xi^{\mathrm{T}} \mathrm{j}\left(\xi \boldsymbol{\omega}_{\mathbf{s}}\right) \hat{\mathbf{f}}^{\mathrm{T}}\left(\xi \boldsymbol{\omega}_{\mathbf{s}}, \omega_{t}\right) \xi-\xi^{\mathrm{T}} \hat{\mathbf{f}}\left(\xi \boldsymbol{\omega}_{\mathbf{s}}, \omega_{t}\right) \mathrm{j}\left(\xi \boldsymbol{\omega}_{\mathbf{s}}\right)^{\mathrm{T}} \xi \\
& =\mathcal{F}\left(\xi^{\mathrm{T}} \operatorname{curl} \mathbf{f}(\xi \mathbf{s}, t) \xi\right) . \\
\mathcal{F}\left\{\partial \mathrm{R}_{\xi} \mathbf{f}\right\} & =\xi^{\mathrm{T}} \mathrm{j} \omega_{t} \hat{\mathbf{f}}\left(\xi \boldsymbol{\omega}_{\mathbf{s}}, \omega_{t}\right)=\mathcal{F}\left\{\mathrm{R}_{\xi} \partial_{t} \mathbf{f}\right\} .
\end{aligned}
$$

Taking magnitudes using $\left|\xi^{\mathrm{T}} \mathbf{f}\right|=|\mathbf{f}|$ and $\left|\xi^{\mathrm{T}} \mathbf{f} \xi\right|=|\mathbf{f}|$ and with the change of variable $\mathbf{u}=\xi \mathbf{s}$, with $\operatorname{du}=|\operatorname{det} \xi| \mathrm{d} \mathbf{s}=\mathrm{d} \mathbf{s}$, we arrive at the desired result.

\subsection{Discretization and Minimization Algorithm}

The discretized flow is expressed by $\mathbf{f}[\mathbf{k}, n]$ over $\mathcal{K} \times \mathcal{N} \subset \mathbb{Z}^{3} \times \mathbb{Z}$ where $\mathcal{K}$ and $\mathcal{N}$ are the bounded spatial and temporal indexing sets. We discretize the partial derivatives involved in the definition of curl, divergence, and time derivative by using the finite-difference operators

$$
\delta_{i}: \mathbf{f} \mapsto \mathbf{f}-\mathbf{f}\left[\cdot-\mathbf{e}_{i}\right],
$$

where $\mathbf{e}_{i}, i=1, \ldots, 4$, denotes the $i$ th standard unit vector in $\mathbb{R}^{4}$. We use $\|\cdot\|_{p}$ notation for both the scalar and vector $\ell_{p}$ norms (for vectors, it corresponds to the scalar $\ell_{p}$ norm of the magnitude of the vector field [6]).

Then, by considering a quadratic fidelity term, we arrive at the discrete formulation of our objective functional given by

$$
\mathcal{J}_{\delta}(\mathbf{f} ; \mathbf{y})=\frac{1}{2}\|\mathbf{f}-\mathbf{y}\|_{2}^{2}+\mathcal{R}_{\delta}^{s}(\mathbf{f})+\mathcal{R}_{\delta}^{t}(\mathbf{f}),
$$

where the discrete spatial and temporal regularizers, respectively, are given by

$$
\begin{aligned}
& \mathcal{R}_{\delta}^{s}(\mathbf{f})=\lambda_{c} \sum_{n \in \mathcal{N}}\left\|\operatorname{curl}_{\delta} \mathbf{f}_{n}\right\|_{1}+\lambda_{d} \sum_{n \in \mathcal{N}}\left\|\operatorname{div}_{\delta} \mathbf{f}_{n}\right\|_{1} \\
& \mathcal{R}_{\delta}^{t}(\mathbf{f})=\lambda_{t}\left\|\partial_{t, \delta} \mathbf{f}\right\|_{2}^{2},
\end{aligned}
$$

with $\mathbf{f}_{n}=\left(f_{n, 1}, f_{n, 2}, f_{n, 3}\right)$ being the $n$th volume for some $n \in \mathcal{N}$. We discretize the curl and divergence operators as

$$
\begin{aligned}
\operatorname{curl}_{\delta} \mathbf{f}_{n} & =\left(\delta_{3} f_{n, 2}-\delta_{2} f_{n, 3}, \delta_{1} f_{n, 3}-\delta_{3} f_{n, 1}, \delta_{2} f_{n, 1}-\delta_{1} f_{n, 2}\right) \\
\operatorname{div}_{\delta} \mathbf{f}_{n} & =\delta_{1} f_{n, 1}+\delta_{2} f_{n, 2}+\delta_{3} f_{n, 3} .
\end{aligned}
$$

The discretized version of the time derivative $\partial_{t, \delta}$ in (8) is given by $\partial_{t, \delta}: \mathbf{f} \mapsto\left(\delta_{4} f_{1}, \delta_{4} f_{2}, \delta_{4} f_{3}\right)$.

In the sequel, we shall derive a minimization algorithm for the convex optimization problem

$$
\mathbf{f}^{\star}=\underset{\mathbf{f}}{\operatorname{argmin}} \mathcal{J}_{\delta}(\mathbf{f} ; \mathbf{y}) .
$$

Definition 1. The proximity operator associated to the functional $\mathcal{R}_{\delta}$ is defined as

$$
\operatorname{prox}_{\mathcal{R}_{\delta}}(\mathbf{y})=\underset{\mathbf{x}}{\operatorname{argmin}} \mathcal{R}_{\delta}(\mathbf{x})+\frac{1}{2}\|\mathbf{x}-\mathbf{y}\|_{2}^{2} .
$$

Hence, we formally specify the solution of (9) as $\mathbf{f}^{\star}=$ $\operatorname{prox}_{\mathcal{R}_{\delta}^{s}+\mathcal{R}_{\delta}^{t}}(\mathbf{y})$ which requires one to compute the proximity operator of $\mathcal{R}_{\delta}^{s}+\mathcal{R}_{\delta}^{t}$. At this point, we apply a Dykstra-like splitting [10] and compute it iteratively by using the individual proximities associated to $\mathcal{R}_{\delta}^{s}$ and $\mathcal{R}_{\delta}^{t}$. The final scheme for (9) is given in Algorithm 1.

The good news is that the proximity operator of $\mathcal{R}_{\delta}^{s}$ is separable and can be implemented in parallel. Basically, at iteration $t$, one needs to solve the nonsmooth problem

$$
\min _{\mathbf{x}} \frac{1}{2}\left\|\mathbf{x}-\left(\mathbf{f}_{n}^{(t)}+\mathbf{p}_{n}^{(t)}\right)\right\|_{2}^{2}+\lambda_{c}\left\|\operatorname{curl}_{\delta} \mathbf{x}\right\|_{1}+\lambda_{d}\left\|\operatorname{div}_{\delta} \mathbf{x}\right\|_{1}
$$

for all $n \in \mathcal{N}$. To compute (10), we use our recent algorithm [8] which relies on Legendre-Fenchel duality arguments. Per contra, the proximity operator of $\mathcal{R}_{\delta}^{t}$ is smooth and can be addressed by linear solvers such as conjugate gradient (CG).

We conclude this section with some remarks regarding the optimization algorithm. We first note that the sequence $\left(\mathbf{f}^{(t)}\right)_{t \rightarrow \infty}$ generated by Algorithm 1 converges to $\mathbf{f}^{\star}$ under the minimal feasibility condition $\operatorname{dom} \mathcal{R}_{\delta}^{s} \cap \operatorname{dom} \mathcal{R}_{\delta}^{t} \neq \emptyset[10]$. However, in practice, it is recommended to increase the number of inner proximal iterations as the outer iterations proceed [11]. We have opted for this solution as an effective method to improve convergence. 


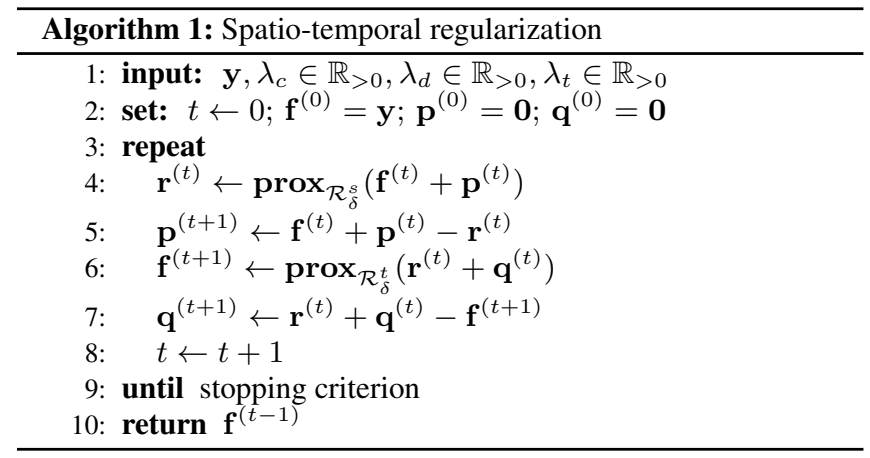

\section{NUMERICAL EXPERIMENTS}

\subsection{Simulated Flow}

A 3D whole-heart MRI dataset was acquired on a 3T clinical MRI scanner (MAGNETOM Trio, Siemens AG, Healthcare Sector, Erlangen, Germany) with a 3D radial trajectory implementing a spiral phyllotaxis pattern [12] that is adapted to self-navigation. The fatsaturated balanced steady-state free precession (bSSFP) measurement was segmented and ECG-triggered. The acquisition window was set to mid-diastole. The following acquisition parameters were used: TR/TE 3.1/1.56 ms, FOV $(220 \mathrm{~mm})^{3}$, matrix $192^{3}$, acquired voxel size $(1.15 \mathrm{~mm})^{3}$, flip angle $70^{\circ}$, and receiver bandwidth $898 \mathrm{~Hz} / \mathrm{Px}$. Throughout the acquisition, a total number of 11687 radial readouts (approximately 20\% radial undersampling) were recorded in free breathing in 377 heartbeats with $100 \%$ scan efficiency.

The complete dataset was processed with the ITK-SNAP software in order to segment a simplified (excluding carotid arteries) 3D geometry of the human aorta. After segmentation, a Laplacian surface smoothing was performed in the Meshlab (3D-CoForm project) environment in order to obtain an eligible wall geometry for computational fluid dynamics. The smoothed STL surface mesh was imported in the ANSYS ${ }^{\circledR}$ ICEM CFD ${ }^{\mathrm{TM}}$ software for the generation of the computational mesh.

Blood was considered to be a Newtonian fluid with density 1040 $\mathrm{Kg} / \mathrm{m}^{3}$ and dynamic viscosity equal 0.004 Pa.s. The inflow boundary was defined as volumetric flow (see Figure 1(a)). The walls were described by the no-slip condition and the solution assumed laminar flow everywhere in the flow field. Since the inflow is time-dependent, the transient solver of ANSYS ${ }^{\circledR} \mathrm{CFX}^{\circledR}$ was used to solve the NavierStokes equations. Four complete cycles were simulated in order to achieve good convergence not only in the spatial, but also in the time dimension. Results were exported every 0.05 of a second and scaled to an Euclidean grid by using linear interpolation. We used ParaView (Kitware Inc.) for vector glyph illustrations.

Table 1. Comparison of denoising algorithms.

\begin{tabular}{l|c|c}
\hline \hline Regularization & Input SNR $[\mathrm{dB}]$ & SNR improvement $[\mathrm{dB}]$ \\
\hline \multirow{2}{*}{ Spatial-only } & 0 & 13.38 \\
& 10 & 10.49 \\
Spatio-temporal & 0 & $\mathbf{1 4 . 4 9}$ \\
& 10 & $\mathbf{1 0 . 9 3}$ \\
\hline
\end{tabular}

The simulated flows were degraded by different levels of additive white Gaussian noise. Spatial-only regularization, with 50 iterations, was then applied to the noisy field where the parameters $\lambda_{c}$ and $\lambda_{d}$ were optimized using an oracle. This method was contrasted with our spatio-temporal method with 10 outer iterations. Inner proximal iterations-starting from 50-were increased by 10 after every outer iteration. To investigate the effect of temporal regularization, we used

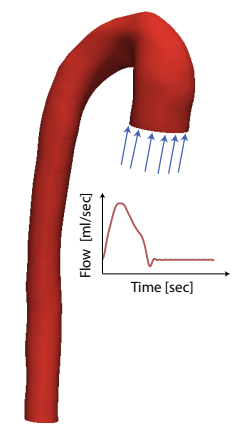

(a)

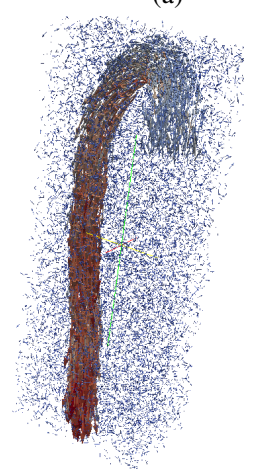

(c)

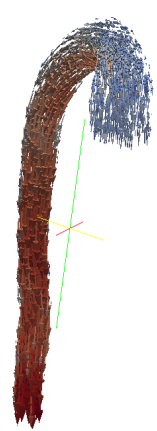

(b)

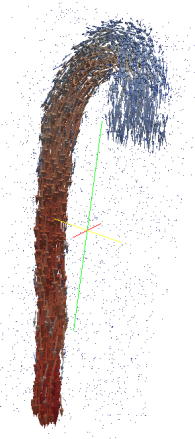

(d)
Fig. 1. Denoising of simulated blood flow at $t=0.1 \mathrm{sec}$ : (a) generated aorta geometry; (b) original blood flow; (c) noisy flow; (d) denoised blood flow by the proposed method.

the previously obtained $\lambda_{c}$ and $\lambda_{d}$ and only optimized $\lambda_{t}$.

The results are summarized in Table 1 . Spatio-temporal regularization is seen to be superior to its spatial-only alternative in terms of SNR for the noise levels considered in the simulations.

\subsection{D Phase-Contrast MRI}

As supplement to our in silico experiments, we considered a multidirectional phase-contrast MRI dataset in the region between the proximal and thoracic aorta of a 25 -year-old healthy male volunteer. PC MRI data was acquired with a sagittal oblique 3D slab covering the entire aorta, using a navigator-gated, ECG-gated RF-spoiled gradient echo (GRE) sequence. The dataset was acquired on a 3T clinical MR scanner (MAGNETOM Trio, Siemens AG, Healthcare Sector, Erlangen, Germany). The sequence was motion compensated and the following imaging parameters were used: TR/TE 5.2/2.59; flip angle $15^{\circ}$; velocity encoding $150 \mathrm{~cm} / \mathrm{sec}$; matrix $224 \times 138 \times 24$; field of view $450 \mathrm{~mm}$, and acquired voxel size $2.0 \mathrm{~mm}^{3}$. Pathline visualizations are made by using GT-Flow (Gyrotools LLC) software.

Based on the near incompressibility (thus almost zero divergence) of the blood flow, we set $\lambda_{d}>\lambda_{c}$. For $\lambda_{t}$, we used the parameter obtained from the simulated flow experiment of $10 \mathrm{~dB}$ input SNR. The algorithm was again run with 10 iterations with the same inner iteration strategy as in our previous experiments.

A qualitative examination of our results showed that the zerodivergence assumption resulted in a great reduction of artifacts and finer visualization of along the whole cardiac cycle. Furthermore, the visualization of the helical flow patterns was improved as a result of the assumption of smoothly varying flow. Based on these two aspects, the regularized data were of substantially better quality compared to those from the initial MRI recordings. 


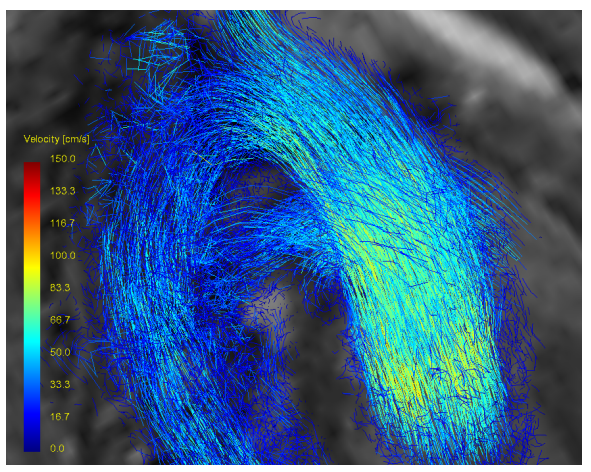

(a)

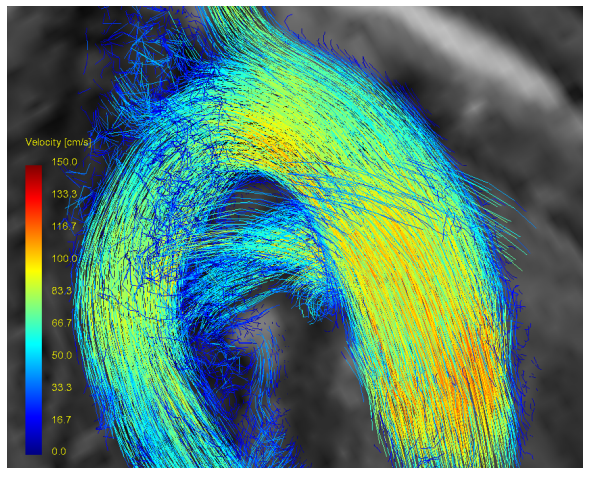

(c)

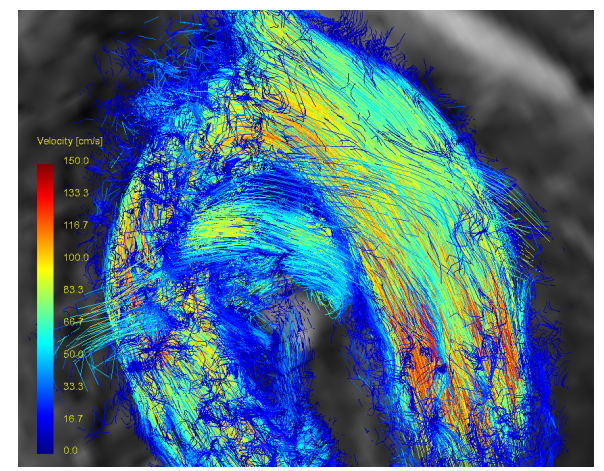

(b)

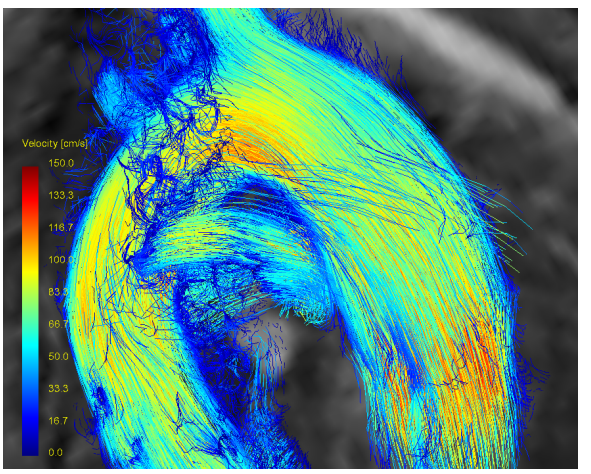

(d)

Fig. 2. Phase contrast MRI: Initial data at (a) $t=31.50 \mathrm{~ms}$, (b) $\mathrm{t}=136.00 \mathrm{~ms}$; Data after regularization at (c) $\mathrm{t}=31.50 \mathrm{~ms}$, (d) $\mathrm{t}=136.00 \mathrm{~ms}$.

\section{CONCLUSION}

We have proposed a variational method for the regularized reconstruction of 3D+time flow fields where the objective functional is justifiable from a physical and mathematical point of view. We have applied a proximal-splitting algorithm for solving the problem and demonstrated improved denoising performance for a simulated blood flow in the human aorta. We have also illustrated that the proposed method has been able to reduce artifacts in a real phase-contrast MRI dataset and enhance the visualisation of the secondary flow patterns in the aorta.

\section{REFERENCES}

[1] M. Markl, A. Frydrychowicz, S. Kozerke, M. Hope, and O. Wieben, "4D flow MRI," J. Magn. Reson. Imaging, vol. 36, no. 5, pp. 1015-1036, 2012.

[2] A. Frydrychowicz, R. Arnold, D. Hirtler, C. Schlensak, Aurelien F. Stalder, J. Hennig, M. Langer, and M. Markl, "Multidirectional flow analysis by cardiovascular magnetic resonance in aneurysm development following repair of aortic coarctation," J. Cardiovasc. Magn. Reson., vol. 10, no. 1, pp. 30, 2008.

[3] O. Vardoulis, E. Coppens, B. Martin, P. Reymond, P. Tozzi, and N. Stergiopulos, "Impact of aortic grafts on arterial pressure: A computational fluid dynamics study," Eur. J. Vasc. Endovasc. Surg., vol. 42, no. 5, pp. 704-710, 2011.

[4] D. Suter and F. Chen, "Left ventricular motion reconstruction based on elastic vector splines," IEEE Trans. Med. Imaging, vol. 19, no. 4, pp. 295-305, 2000.
[5] M. Arigovindan, M. Sühling, C. Jansen, P. Hunziker, and M. Unser, "Full motion and flow field recovery from echo doppler data," IEEE Trans. Med. Imaging, vol. 26, no. 1, pp. 31-45, 2007.

[6] P. D. Tafti and M. Unser, "On regularized reconstruction of vector fields," IEEE Trans. Image Process., vol. 20, no. 11, pp. 3163-3178, 2011.

[7] P. D. Tafti, R. Delgado-Gonzalo, A. F. Stalder, and M. Unser, "Variational enhancement and denoising of flow field images," in Proc. 8th IEEE Int. Symp. Biomed. Imaging (ISBI'11), 2011, pp. 1061-1064.

[8] E. Bostan, P. D. Tafti, and M. Unser, "A dual algorithm for $\mathrm{L}_{1}$ regularized reconstruction of vector fields," in Proc. 9th IEEE Int. Symp. Biomed. Imaging (ISBI'12), 2012, pp. 1579-1582.

[9] W. W. Nichols, M. F. O'Rouke, and C. Vlachopoulos, McDonald's Blood Flow in Arteries: Theoretical, Experimental and Clinical Principles, Hodder Arnold, 2011.

[10] P. L. Combettes, "Iterative construction of the resolvent of a sum of maximal monotone operators," J. Convex Anal., vol. 16, no. 4, pp. 727-748, 2009.

[11] M. Schmidt, N. L. Roux, and F. Bach, "Convergence rates of inexact proximal-gradient methods for convex optimization," in Proc. 25th Annu. Conf. Neural Inf. Process. Syst. (NIPS'11), 2011, pp. 1458-1466.

[12] D. Piccini, A. Littmann, S. N.-Vallespin, and O. M. Zenge, "Spiral phyllotaxis: The natural way to construct a 3D radial trajectory in MRI," Magn. Reson. Med., vol. 66, no. 4, pp. 1049-1056, 2011. 